\title{
Coupling effect of surfactant sprays and gas jet on inhalable particle agglomeration
}

\author{
Yali Liu ${ }^{\mathrm{a}}$, Deshuai Sun ${ }^{\mathrm{b} *}$, Tingting Qin $^{\mathrm{c}}$ and Zhongyi Zhang ${ }^{\mathrm{d}}$ \\ College of Chemical Engineering, Qingdao University, Qingdao, 266071, China

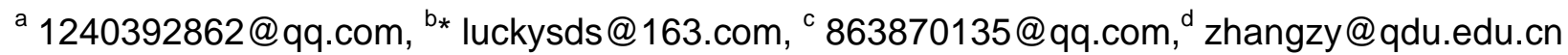

Keywords: Inhalable particle; agglomeration; surfactant; gas jet; coupling effect

Abstract. Inhalable particles are harmful to public health and cause serious environmental problems. In this paper, surfactant sprays and gas jet were introduced into the agglomeration chamber to encourage the agglomeration of inhalable particles. The coupling effect of double gas jet and surfactant spray could remove $51.3 \%$ of inhalable particles. The mass mean diameter increased from $3.5 \mu \mathrm{m}$ to $5.1 \mu \mathrm{m}$, $5.7 \mu \mathrm{m}$ and $8.7 \mu \mathrm{m}$ under the action of surfactant spry, double jet and the coupling effect, respectively. Surfactant species had a remarkable influence on particle agglomeration. Non-ionic surfactant, OP-10, could remove more than $50 \%$ of inhalable particles. Increasing the concentration of surfactant agents could result in the incremental removal efficiency of inhalable particles. In double gas jet experiment, when gas jet located in asymmetrical sites, more inhalable particles were removed.

\section{Introduction}

In China, coal is the most abundant fossil fuel resources. The emission of particles from coal-fired plant has gained much attention. At present, $99 \%$ particles in flue can be captured by conventional filters, such as electrostatic precipitators, cyclones and wet scrubbers. However, 1\% particles left in flue are inhalable particles with diameter less than $10 \mu \mathrm{m}$, which are difficult to be separated by conventional filters. They are emitted into atmospheric environment. These inhalable particles in air can enter the human blood circulation by penetrated deeply into the alveolus of respiratory system, which can cause cardiovascular disease and chronic bronchitis [1]. The inhalable particles do great harm to human health because they are commonly enriched with heavy metal (e.g. selenium, lead, chromic) and noxious agents (e.g. bacteria, dioxin) [2]. Therefore, it is urgent to explore a new technology to reduce the inhalable particles in flue.

Due to their very small diameter, inhalable particles have strong adhesive forces, such as van der wall forces, electrostatic forces, and capillary forces. When they collide with another large particle, they can form a larger agglomeration under the action of adhesive forces. Agglomeration technology is regarded as a promising aerosol preconditioning. The adhesive force can be intensified by adsorbing surfactant agent [3]. And the collision between particles can be strengthened by turbulent jet [4,5].

In this paper, surfactant was used to wet particle surface and reduce interfacial force. Gas jet was introduced in agglomeration chamber to form the local turbulent flow. The coupling effect of surfactant spray and gas jet on inhalable particles preconditioning were investigated.

\section{Experimental sections}

Experimental material. The inhalable particle sources were coal-fired fly ash particles, which were collected from the electrostatic precipitator in Qingdao power plants. The moisture content of fly ash was $0.78 \%$, and the density was $1838 \mathrm{~kg} / \mathrm{m}^{3}$. The initial size distribution of the fly ash was measured using the Rise-2002 laser particle size analyzer (Rise Science Co., Ltd.), and the median aerodynamic diameter was $14.36 \mu \mathrm{m}$.

Surfactant agents included alkylphenol ethoxylates (OP-10, non-ionic surfactant), twelve benzene sulfonic acid sodium (SDBS, anionic surfactant), cetyltrimethyl ammonium bromide (CTB, cation surfactant). They were bought from chemical factory and used without purification. 
Experimental setup. As shown in Fig.1, the experimental setup composed of a fluidized bed aerosol generator, a gas jet system, a spray system, a measurement apparatus, and a particle agglomeration chamber. The inner diameter of the fluidized bed aerosol generator was $54 \mathrm{~mm}$, and the length was 600 $\mathrm{mm}$. The particle agglomeration chamber $(\Phi 116 \times 600 \mathrm{~mm})$ was a round pip, and two ends of the tube were sealed by a plate of polymethyl methacrylate. The aerosol exitings with inner diameter of $20 \mathrm{~mm}$ were fixed on the center of the plate.

Several inlets were open symmetrically on the opposite of the agglomeration chamber with inner diameter of $10 \mathrm{~mm}$. The distance was $100 \mathrm{~mm}, 200 \mathrm{~mm}$ and $300 \mathrm{~mm}$ from the upper aerosol inlet. The spray system comprised of a Compmist40-105-000 pneumatic atomizer and an atomizer cup, which could generate droplet with diameter of $25.2 \mu \mathrm{m}$ (Dv90). A round spray nozzle with inner diameter of $8 \mathrm{~mm}$ and jet nozzles with inner diameter of $1.4 \mathrm{~mm}$ were fixed vertically on the predetermined inlets of the chamber.

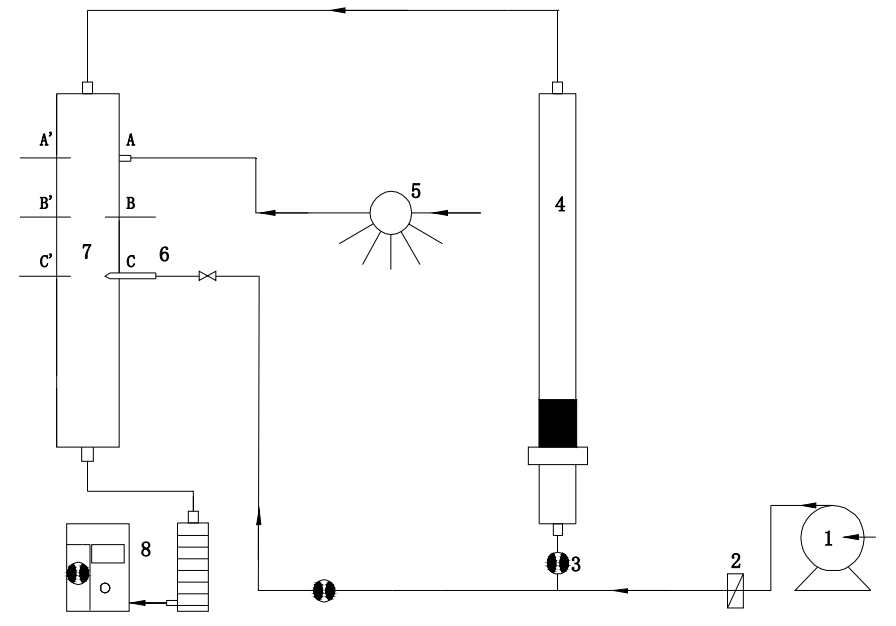

1. Compressor 2. HEPA filter 3. Rotameter

4. Fluidized bed aerosol generator

5. Atomizer 6. Jet nozzle

7. Agglomeration chamber 8. Cascade impactor

Fig. 1. Particle agglomeration experimental setup

A FA-3 aerosol cascade impactor (Kangjie Instrument Institute) was used to collect aerosol samples with diameter in the range from $0.43 \mu \mathrm{m}$ to $10 \mu \mathrm{m}$. Table 1 showed the cut point diameters of a cascade impactor.

Table 1. Cut point diameters of the cascade impactor

\begin{tabular}{lccccccccc}
\hline Stage & 0 & 1 & 2 & 3 & 4 & 5 & 6 & 7 & 8 \\
\hline Particle diameter $[\mu \mathrm{m}]$ & $9.0-10$ & $5.8-9.0$ & $4.7-5.8$ & $3.3-4.7$ & $2.1-3.3$ & $1.1-2.1$ & $0.65-1.1$ & $0.43-0.65$ & $0-0.43$ \\
Pore diameter $[\mathrm{mm}]$ & 2.25 & 1.89 & 0.91 & 0.71 & 0.53 & 0.34 & 0.25 & 0.25 & Membrane \\
\hline
\end{tabular}

Experimental procedure. Air filtered by a high-efficiency particulate filter was supplied to fluidize the bed. Fly ash was dispersed to form aerosol, which was subsequently entrained into the chamber. The aerosol velocity was $0.22 \mathrm{~m} / \mathrm{s}$. The surfactant solution was atomized and introduced into the chamber through the location of A ( Fig.1). Its flow was controlled about $0.2-0.3 \mathrm{~mL} / \mathrm{min}$. The velocities of jet gas were $22.6 \mathrm{~m} / \mathrm{s}$ and $11.3 \mathrm{~m} / \mathrm{s}$ for single jet and double jet, respectively. The location of single gas jet was B' or C', while the site was A'-C, B'-C or B'-B for double jet. In this test, all experiments were performed at ambient conditions and all data were the average of three runs at an 8-hour continuous operation.

\section{Results and discussion}

In agglomeration process, the total particle removal efficiency was the percentage decrement in total particle mass over time intervals.

Effect of gas jet and surfactant spray on particle removal efficiency. The gas jet facilitated the collision between the particles. After that the surfactant increased the adhesion among particles to form large agglomeration. The effect of gas jet and surfactant spray on particle removal efficiency was showed in Fig.2. Agglomeration increased the diameter of aggregates. The mass mean diameter was 
$3.5 \mu \mathrm{m}$ for initial inhalable particles. These values increased to $5.1 \mu \mathrm{m}, 5.7 \mu \mathrm{m}$ and $8.7 \mu \mathrm{m}$ under the action of surfactant spray, double jet and the coupling effect, respectively. It also showed that the coupling field could improve significantly the removal sufficiency to $51.3 \%$ from $36.1 \%$ of double gas jet and $25.4 \%$ of surfactant spray.

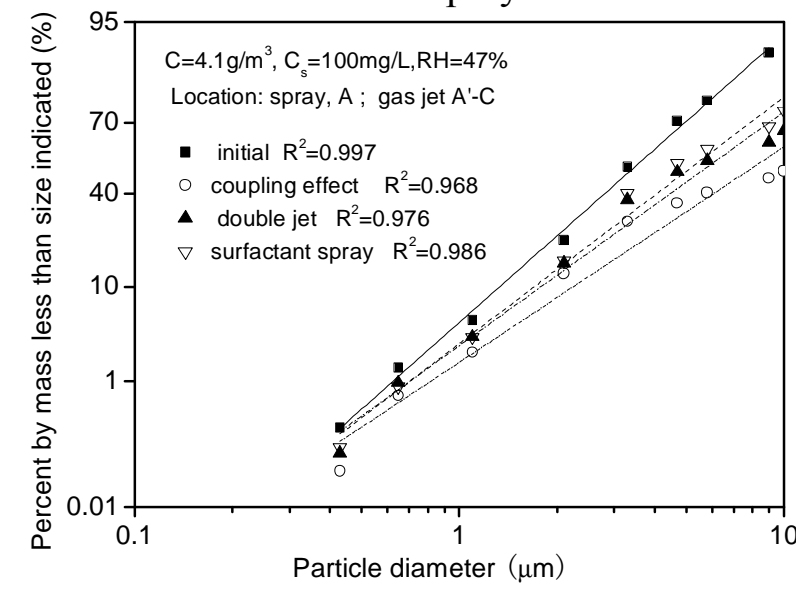

Fig. 2 Effect of gas jet and surfactant spray on particle removal efficiency

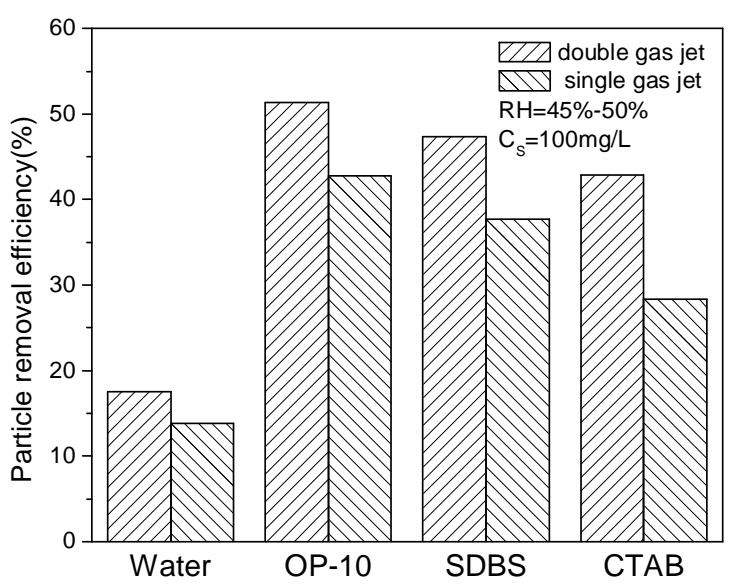

Fig. 3 Effect of surfactant species on particle removal efficiency

Effect of surfactant species on removal efficiency. Surfactant could wet the surface of particles and increase adhesion between particles. Fig. 3 showed the removal effect of different surfactant species. The addition of surfactant could increase the removal efficiency of inhalable particles. As comparison, Non-ionic surfactant, OP-10, could remove more than $50 \%$ of inhalable particles. Anionic surfactant, SDBS, and cation surfactant, CTAB, could decrease $47.4 \%$ and $42.87 \%$ of particle, respectively. These results could be due to the different charge of surfactant agents. The $\mathrm{pH}$ of zero charge of the fly ash was $9.3[6,7]$ while the $\mathrm{pH}$ of surfactant solution was 6-8. When the ionic surfactant adsorbed and aggregated on the surface of fly ash, there would be some charge on the particles. The electrostatic repulsion between the same charge would hinder the further agglomeration among particles. In addition, the effect of double gas jet was superior to that of single gas jet.

Effect of surfactant concentration on particles distribution. In this experiment, nonionic OP-10 and anionic SDBS were chosen as two topical surfactants to investigate the effect of concentration (Fig.4). When the surfactant spray was introduced into the agglomeration chamber, the mass of particles decreased, especially for particles diameter range from $2 \mu \mathrm{m}$ to $4 \mu \mathrm{m}$. This result indicated that larger agglomeration was forming. More particles were removed with the increment of surfactant concentration. This could be the fact that the active site increased on the surface of particles with increasing the surfactant concentration [8].
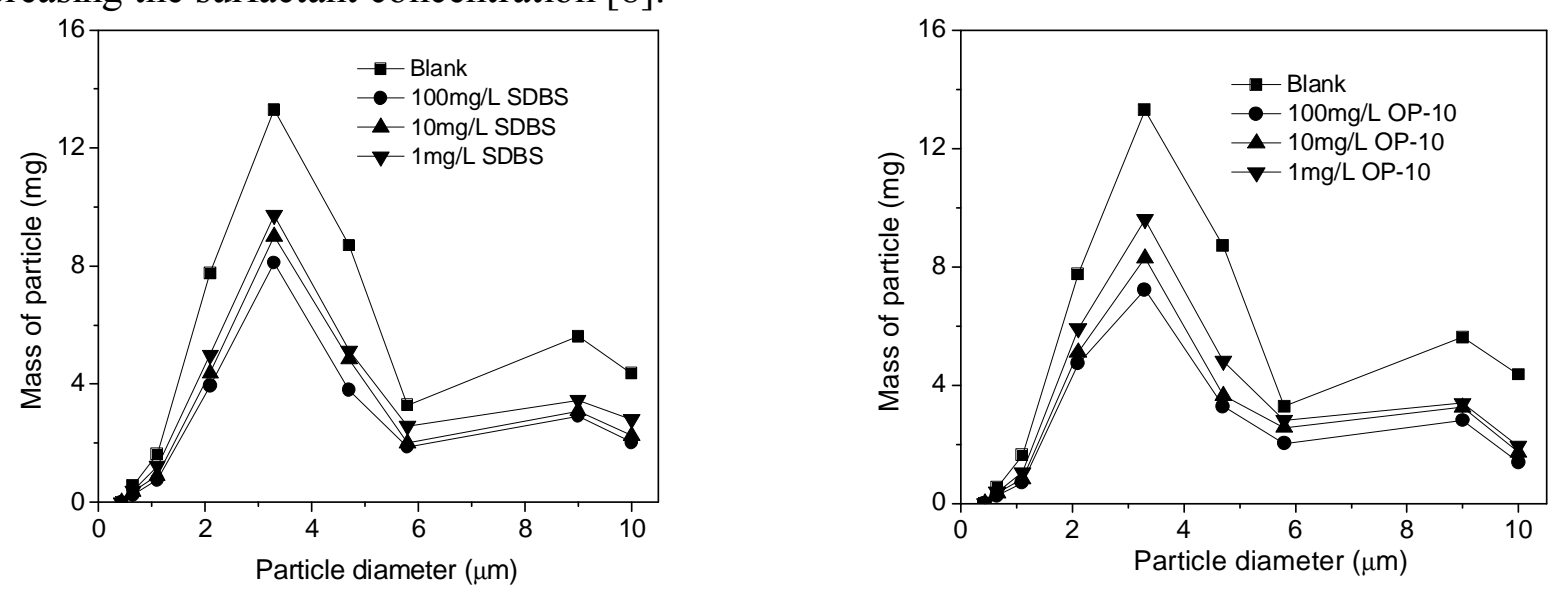

Fig. 4 Effect of surfactant concentration on particle distribution

Effect of jet injection site on particle removal efficiency. The jet gas could generate the local turbulent field in the particle agglomeration chamber to increase the chance of collision between particles. Fig. 5 revealed the effect of the jet injection site on particle removal efficiency. In double gas 
jet experiment, the removal efficiencies were $51.3 \%, 43.33 \%$ and $31.85 \%$ for the location of A-C', B-C' and B-B', respectively. When gas jet located in asymmetrical sites, more inhalable particles were removed. This could be explained by the energy loss in the symmetrical gas jet. As the distance between the opposite sides of the double jet pip increased, surfactant agent could wet completely the surface of particles to increase adhesion force among particles.

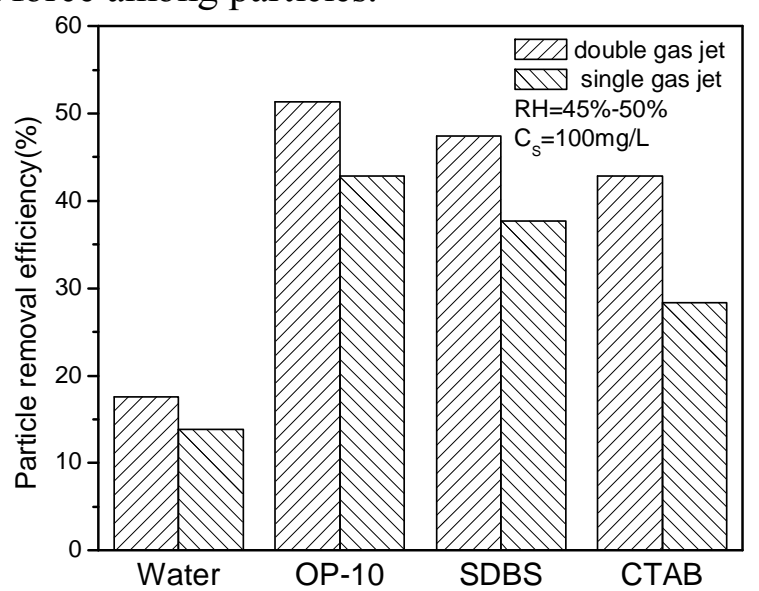

Fig.5. Effect of jet injection sites on particle removal efficiency

\section{Conclusions}

The conclusions could be derived from the investigation:

1) Agglomeration shifted the particles to large aggregates. The mass mean diameter increased from $3.5 \mu \mathrm{m}$ to $5.1 \mu \mathrm{m}, 5.7 \mu \mathrm{m}$ and $8.7 \mu \mathrm{m}$ under the action of surfactant spry, double jet and the coupling effect, respectively. The coupling effect of double gas jet and surfactant spray could remove $51.3 \%$ of inhalable particles.

2) Non-ionic surfactant, OP-10, could remove more than $50 \%$ of inhalable particles. Anionic surfactant, SDBS, and cation surfactant, CTAB, could decrease $47.4 \%$ and $42.87 \%$ of particle. The removal efficiency of inhalable particles increased with the increment of surfactant concentration. In double gas jet experiment, when gas jet located in asymmetrical sites, more inhalable particles were removed. The removal efficiencies were in range from $31.85 \%$ to $51.3 \%$.

\section{Acknowledgment}

The authors wish to acknowledge the financial support from National Natural Science Foundation of China (Contract No. 21206080) and from Postdoctoral Innovation Fund of Shandong Province (Contract No. 201203027).

\section{References}

[1]S.S. Park, A.S. Wexler.Particle deposition in the pulmonary region of the human lung: A semi-empirical model of single breath transport and deposition, J. Aero. Sci. 38 (2007) 228-245. [2]Q.Guo, Z.Yang, J. Zhang. Influence of a combined external field on the agglomeration of inhalable particles from a coal combustion plant, Powder Tech. 227 (2012) 67-73.

[3]D. Sun, L. Fang, Y. Liu. Influence of surfactant sparys on agglomeration of inhalable particle from coal combustion, Adv. Mater. Res. 1044-1045(2014)344-347

[4]D.Sun, Q.Guo. Effect of Gas Jet on Agglomeration of Inhalable Particles, Int J Chem React Eng, 8 (2010) 4-8.

[5]D. Sun, X. Zhang, L. Fang. Coupling effect of gas jet and acoustic wave on inhalable particle Agglomeration, J. Aero. Sci. 66 (2013) 12-23.

[6] P.C. Faria, J.J. Orfao, M.F. Pereira, Pereira. Adsorption of anionic and cationic dyes on activated carbons with different surface chemistries, Water Res. 38 (2004) 2043-2052. 
[7]D. Sun, X. Zhang, L. Fang, M. Wang. Investigation into wettability of surfactant on fly ash. Annual meeting of Chemical Industry and Engineering Society of China, 2013.

[8]Y. Zhao, J. Zhang, F. Wei. Experimental study on agglomeration of submicron particles from coal combustion, J. Chem. Ind. Eng.(China). 58(2007) 2876-2881. 